\title{
Improved Diagnostic Accuracy in Characterization of Adnexal Masses by Detection of Choline Peak Using 1H MR Spectroscopy in Comparison to Internal Reference at 3 Tesla
}

\author{
Mahrooz Malek ${ }^{1}$, Maryam Pourashraf ${ }^{1 *}$, Mitra Modares Gilani², Masoumeh \\ Gity $^{1}$
}

\begin{abstract}
Background: The aim of this study was to assess the role of the presence of a choline peak in 3 Tesla $1 \mathrm{H}$ magnetic resonance spectroscopy (MRS) for differentiating benign from malignant adnexal masses. Materials and Methods: A total of 46 adnexal masses (23 malignant and 23 benign) underwent $1 \mathrm{H}$ MRS study prior to surgery to assess the presence of choline peak. Results: A choline peak was detected in 16 malignant masses $(69.5 \%)$ and was absent in the other $7(30.5 \%)$. A choline peak was only detected in $6(26 \%)$ of the benign adnexal masses. The presence of an MRS choline peak had a sensitivity of $69.5 \%$, a specificity of $74 \%$, a positive predictive value (PPV) of $72.7 \%$, and a negative predictive value (NPV) of $71 \%$ for diagnosing malignant adnexal masses. A significant difference between the frequency of mean choline peaks in benign and malignant adnexal masses was observed ( $P$ value $<0.01)$. Conclusions: A 1H MRS choline peak is seen in malignant adnexal masses more frequently than the benign masses, and may be helpful for diagnosing malignant adnexal masses
\end{abstract}

Keywords: Adnexal mass - magnetic resonance spectroscopy - choline - malignancy

Asian Pac J Cancer Prev, 16 (12), 5085-5088

\section{Introduction}

Adnexal masses are among the common gynecologic problems. They may be of ovarian or non-ovarian origin. Most of the adnexal masses are benign and only a small number are malignant (Mohaghegh and Rockall, 2012; Kunpalin et al., 2014). Ovarian cancer is a major cause of death from gynecologic malignancies in women (Boyle et al., 2000). Preoperative characterization of adnexal masses and determining the likelihood of malignancy are important in the successful management of these masses (Arikan et al., 2014; Karadag et al., 2014; Tantipalakorn et al., 2014). Although ultrasound is the primary imaging modality, magnetic resonance (MR) imaging provides additional information on the nature of the lesions. Risk of malignancy index (RMI) is a quantitative tool for preoperative risk assessment of adnexal masses (ArunMuthuvel and Jaya, 2014; Simsek et al., 2014; Ozbay et al., 2015). MRI without and with intravenous contrast is required for indeterminate adnexal masses, either with uncertain origin or with intermediate RMI in the range of 25-200 (Mohaghegh and Rockall, 2012).

In the recent years, studies have been carried out to investigate the role of the new functional MR techniques such as dynamic contrast-enhanced MR imaging (DCEMRI), diffusion weighted imaging (DWI) and magnetic resonance spectroscopy (MRS) in distinguishing benign from malignant adnexal masses (Stanwell et al., 2008; Thomassin-Naggara et al., 2008; Takeuchi et al., 2010; Thomassin-Naggara et al., 2011). MRS is a technique which evaluates the biochemical content of tissues. It can provide diagnostic and prognostic information in various diseases of different organs (Swindle et al., 2003; $\mathrm{Oz}$ et al., 2014). One of the most commonly measured metabolites in MRS is choline which is an indicator of cell proliferation and active tumor (Negendank, 1992; Bartella et al., 2006).

The objective of this study was to assess the accuracy of $1 \mathrm{H}$ MRS Choline peak for distinguishing benign versus malignant adnexal masses.

\section{Materials and Methods}

\section{Study subjects}

The study protocol was approved by the institutional review board of the university hospital, and written 
informed consent was taken from all patients. Initially a total of 54 consecutive patients with adnexal masses entered the study. Regarding the low signal-to-noise ratio, the MRS spectra of 8 masses were excluded from the study. Finally, 46 adnexal masses were studied during the study period.

\section{MRI and MRS acquisition}

All patients underwent pelvic MRI and 1H MRS before laparotomic or laparoscopic surgery. The time interval between the MRS study \& surgery was less than one month. All MRI examinations were performed on a 3 Tesla MR unit (Magnetom Avanto; Siemens, Erlangen, Germany) with an eight-channel phased-array pelvic coil. The patients were fasting for at least 6 hours before MRI. The peristaltic motion artifacts were reduced by using intramuscular injection of Hyoscine.

Before MRS, conventional pelvic MRI protocols for the assessment and localization of the adnexal masses

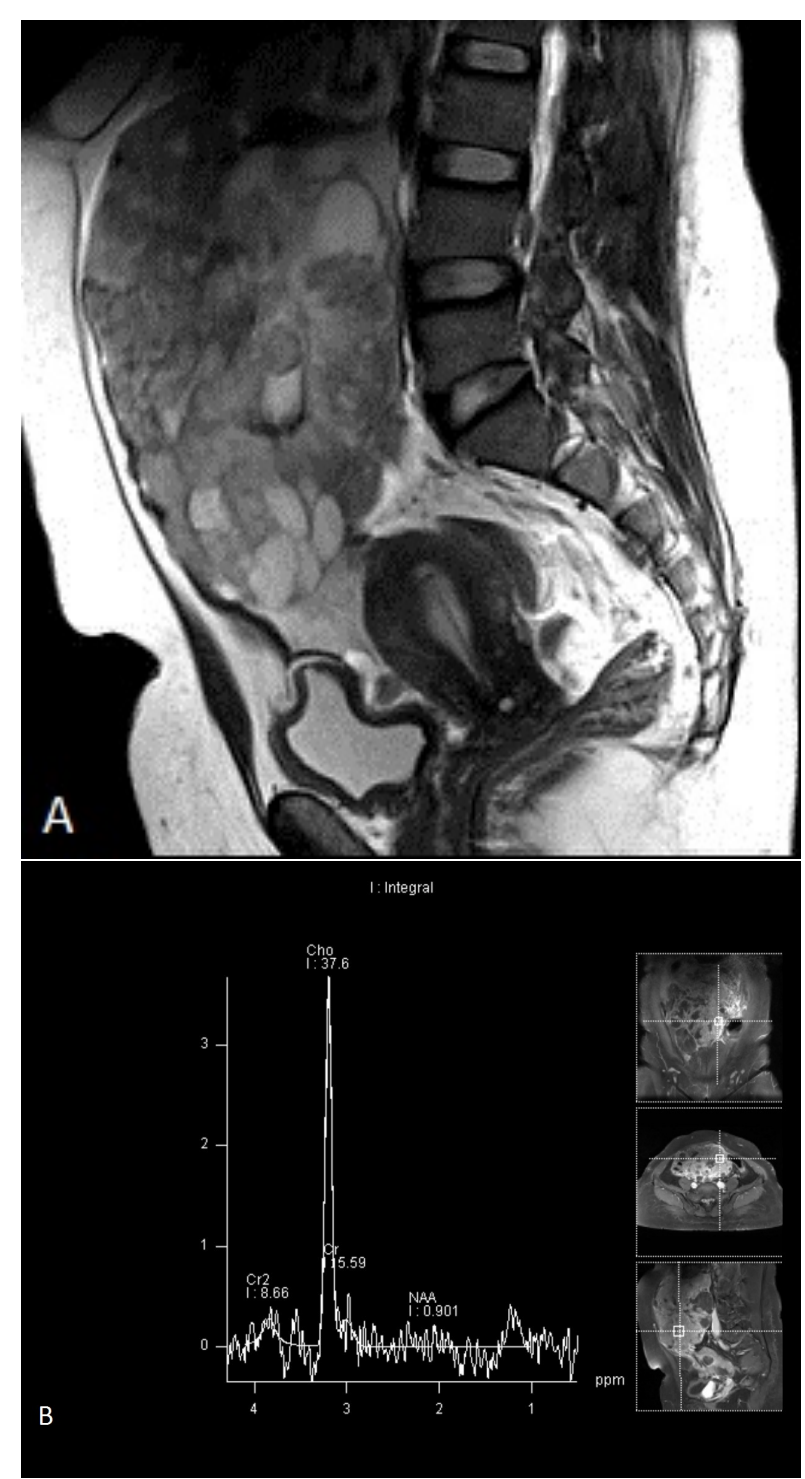

Figure 1. A 38-year-old female with serous cystadenocarcinoma. A) Sagittal fast spin-echo T2weighted image shows a large adnexal mass with solid and cystic components. B) Single-voxel 1H MR spectrum (3 Tesla, PRESS sequence) shows a choline peak were acquired, which included axial, sagittal, and coronal fast spin-echo (FSE) T2-weighted images(repetition time (TR)/echo time (TE), 4200/110 ms; matrix, 256×512; field of view (FOV), $24 \mathrm{~cm}$; slice thickness, $4 \mathrm{~mm}$ ), and axial spin echo T1-weighted images (TR/TE, 450/25 ms; matrix, 224×448; FOV, $24 \mathrm{~cm}$; slice thickness, $4 \mathrm{~mm}$ ). After intravenous administration of $0.1 \mathrm{mmol} / \mathrm{kg}$ body weight of gadoterate meglumine (Dotarem, Guerbet), axial T1-weighted images were obtained again.

Single voxel MRS (SVS) was performed following lesion identification on the unenhanced and enhanced MR images. Coronal, sagittal, and axial contrast-enhanced T1-weighted images were used for the voxel placement. Automated and manual shimming procedures were performed to improve magnetic field homogeneity. The voxel was placed on the enhancing component of the adnexal masses without contamination from adjacent tissues by a radiologist with 6 years of experience in female pelvic MRI (M.M). Voxel size was 2 to $12 \mathrm{~cm} 3$ depending on the size of the solid component. Spectra were obtained using PRESS (point resolved spectroscopy) sequence with TE of $135 \mathrm{~ms}$, TR of $2000 \mathrm{~ms}$, and 8 signal averages .The time of acquisition was 5 minutes. Water suppressed and non-water suppressed acquisition techniques were applied for all patients. The internal

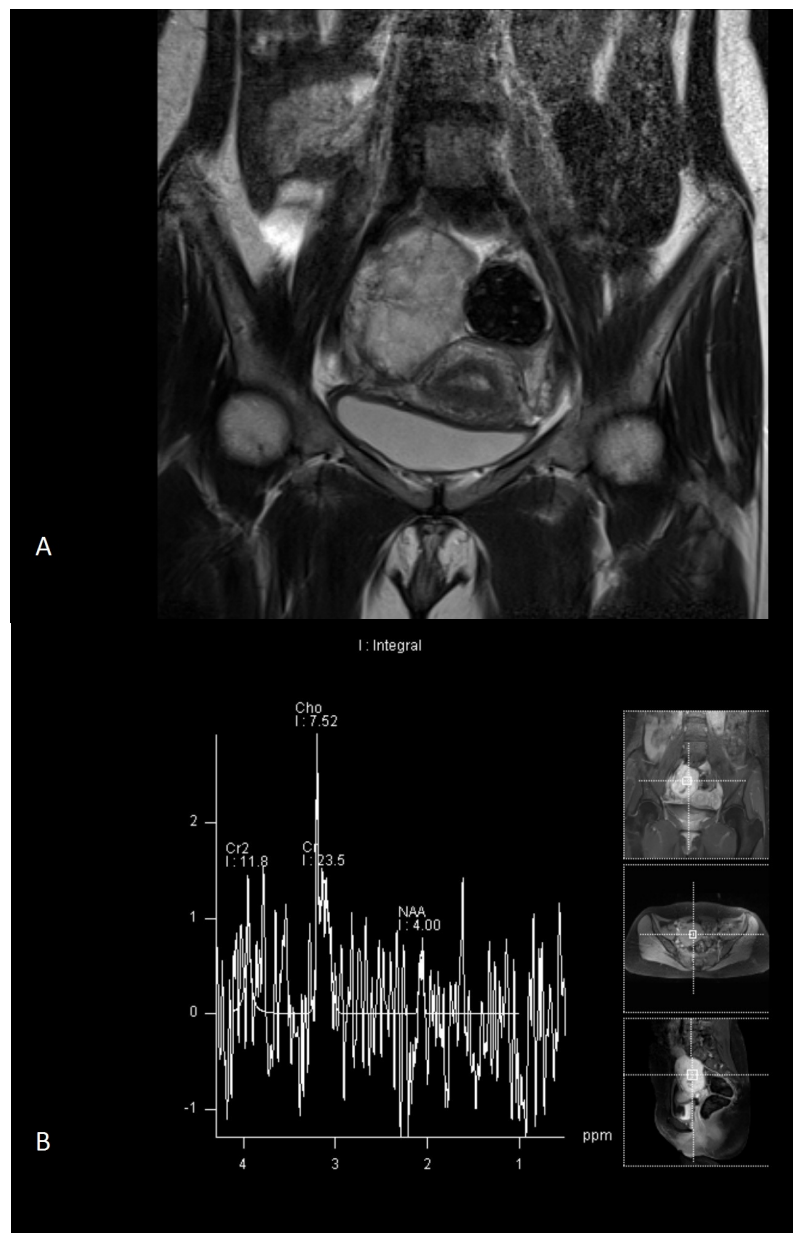

Figure 2.A 28-year-old woman with adnexal metastasis of colon adenocarcinoma. A) Coronal fast spin-echo T2weighted image shows a solid adnexal mass. B) Single-voxel 1H MR spectrum (3 Tesla, PRESS sequence) shows the presence of a choline peak 
reference was psoas muscle. Finally, by measuring the full-width half maximum (FWHM) of water resonance in non-water suppressed acquisition, spectroscopic quality was assessed. Each voxel was evaluated for the presence of a choline peak. Signal to noise ratio of spectrum was considered acceptable if the amplitude of choline peak was 3-times greater than the amplitude of baseline noise.

\section{Statistical analysis}

The surgical pathological findings were used as the reference standard for the assessment of adnexal masses. All analyses were carried out using SPSS version 18 for Windows (SPSS, Chicago, IL, USA). Continuous variables were described as mean \pm SD. Categorical variables were reported as frequency and percentage. Statistical significance for intergroup differences was evaluated by chi-square test. In all tests, a P value $<0.05$ was considered statistically significant.

\section{Results}

In the study period, 46 adnexal masses were evaluated by $1 \mathrm{H}$ MRS. The mean patient age was $34.9 \pm 15$ (range, 14 to 76) years. The time interval between the MRS study $\&$ surgical resection was less than one month. The mean age of the patients with benign versus malignant adnexal

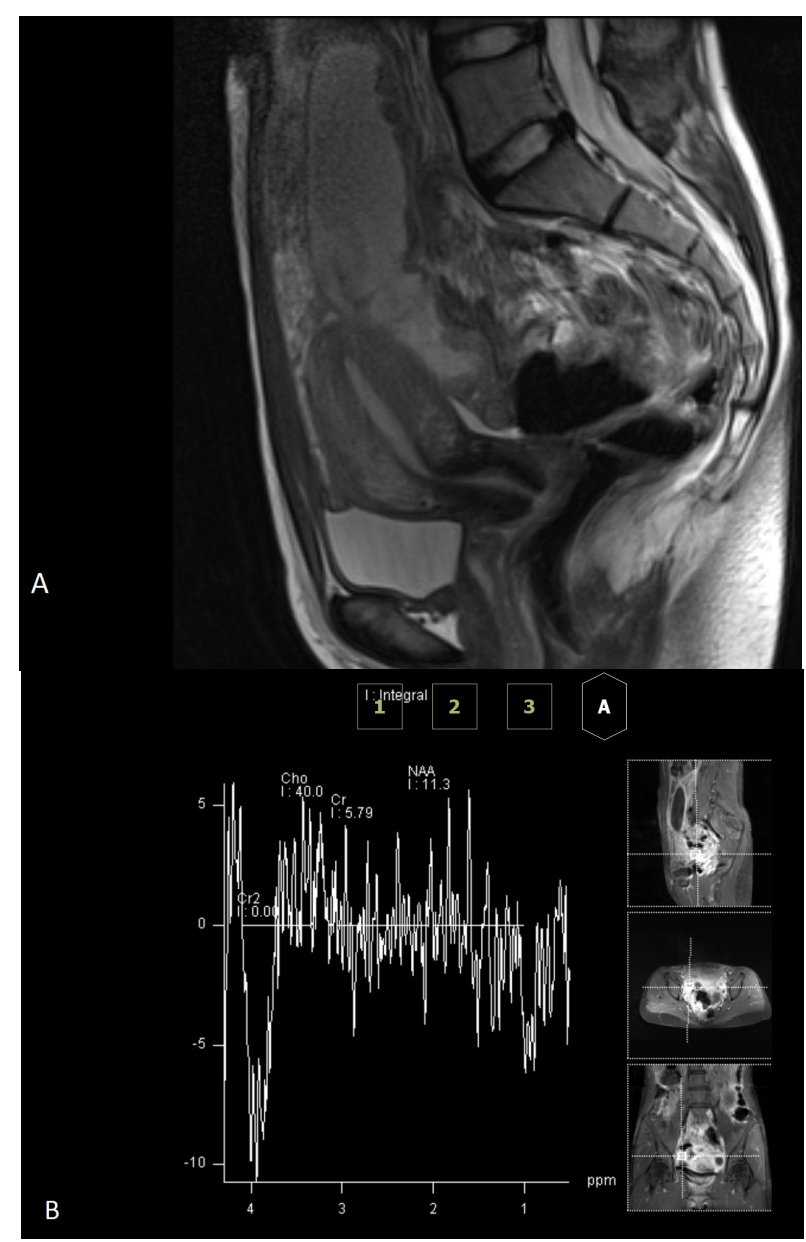

Figure 3. A 16-year-old girl with adnexal tuberculosis. A) Sagittal fast spin-echo T2-weighted image shows a complex adnexal mass. B) Single-voxel 1H MR spectrum (3 Tesla, PRESS sequence) shows absence of choline peak masses was $31.1 \pm 12.1$ and $38.7 \pm 12.3$ years, respectively. Based on the post-operative pathological findings, 23 $(50 \%)$ of the masses were malignant and $23(50 \%)$ were benign. Serous cystadenocarcinoma (9 masses) and mucinous cystadenocarcinoma (5 masses) were the most frequent types of malignant masses. Endometrioma (7 masses) was the most frequent benign adnexal mass.

The results of the $1 \mathrm{H}$ MRS study of the adnexal masses showed that 22 masses (47\%) had a choline peak (Figures 1 and 2). The choline peak was detected in 16 malignant masses $(69.5 \%)$ and was absent in 7 other malignant masses $(30.5 \%)$. The choline peak was detected in 6 (26\%) benign adnexal masses ( 2 adnexal tuberculosis, 2 dermoid cysts, 1 endometrioma, 1 cystadenofibroma). Absence of choline peak was noted in 17 (74\%) of benign masses (Figure 3). Our results revealed that the presence of $1 \mathrm{H}$ MRS choline peak has a sensitivity of $69.5 \%$, a specificity of $74 \%$, a positive predictive value (PPV) of $72.7 \%$, and a negative predictive value (NPV) of $71 \%$ for diagnosing malignant adnexal masses. A significant difference between the frequency of the mean choline peak in benign and malignant adnexal masses was observed ( $\mathrm{P}$ value $<0.01$ ).

\section{Discussion}

Characterization of adnexal masses as benign or malignant, plays an important role in determining the best management strategy. Ultrasound and MRI are the most frequently used imaging modalities for this purpose. In the recent years, the role of the more recent functional MR techniques in the evaluation of adnexal masses has been investigated. One of these techniques is MRS which is a modality complementary to conventional MRI and helps in lesion characterization in different organs.

The present study showed that the 1H MRS choline peak is seen in malignant adnexal masses more frequently than the benign adnexal masses. The presence of $1 \mathrm{H}$ MRS choline peak has a sensitivity of $69.5 \%$, and a specificity of $74 \%$ for diagnosing malignant adnexal masses. In a prior study by Booth et al. (2009) performed at 3 Tesla, choline peak was present in 13 of 14 (93\%) of malignant ovarian masses. But they found no statistically significant difference between choline levels of benign and malignant masses. In another study by Esseridou et al. (Esseridou et al., 2011), 3-dimensional proton MRS was performed in 23 ovarian masses at $1.5 \mathrm{~T}$. Their results showed the presence of choline peak in 17 of 19 malignant masses (sensitivity 89\%) and in 3 of 4 benign masses. They also observed a significant difference between the mean choline peak integral detected within the solid component and that within the cystic component of the ovarian masses $(\mathrm{P}=0.002)$.

The main limitation of the present study was the limited number of cases. Further studies with larger number of cases are needed to confirm these results. Moreover, studies on a larger number of benign and malignant adnexal masses could be helpful in finding an optimal cutoff point of absolute choline concentration for distinguishing benign from malignant adnexal masses.

In conclusion, the results of this study revealed that 


\section{Mahrooz, Malek et al}

the $1 \mathrm{H}$ MRS choline peak is seen in malignant adnexal masses more frequently than the benign masses and the presence of $1 \mathrm{H}$ MRS choline peak is helpful for diagnosing malignant adnexal masses.

\section{References}

Arikan SK, Kasap B, Yetimalar H, et al (2014). Impact of prognostic factors on survival rates in patients with ovarian carcinoma. Asian Pac J Cancer Prev, 15, 6087-94.

Arun-Muthuvel V, Jaya V (2014). Pre-operative evaluation of ovarian tumors by risk of malignancy index, CA125 and ultrasound. Asian Pac J Cancer Prev, 15, 2929-32.

Bartella L, Morris EA, Dershaw DD, et al (2006). Proton MR spectroscopy with choline peak as malignancy marker improves positive predictive value for breast cancer diagnosis: preliminary study. Radiol, 239, 686-92.

Booth SJ, Pickles MD, Turnbull LW (2009). In vivo magnetic resonance spectroscopy of gynaecological tumours at 3.0 Tesla. BJOG, 116, 300-3.

Boyle P, Maisonneuve P, Autier P (2000). Update on cancer control in women. Int J Gynaecol Obstet, 70, 263-303.

Esseridou A, Di Leo G, Sconfienza LM, et al (2011). In vivo detection of choline in ovarian tumors using 3D magnetic resonance spectroscopy. Invest Radiol, 46, 377-82.

Karadag B, Kocak M, Kayikcioglu F, et al (2014). Risk for malignant and borderline ovarian neoplasms following basic preoperative evaluation by ultrasonography, ca125 level and age. Asian Pac J Cancer Prev, 15, 8489-93.

Kunpalin Y, Triratanachat S, Tantbirojn P (2014). Proportion of ovarian cancers in overall ovarian masses in Thailand. Asian Pac J Cancer Prev, 15, 7929-34.

Mohaghegh P, Rockall AG (2012). Imaging strategy for early ovarian cancer: characterization of adnexal masses with conventional and advanced imaging techniques. Radiographics, 32, 1751-73.

Negendank W (1992). Studies of human tumors by MRS: a review. NMR Biomed, 5, 303-24.

Oz G, Alger JR, Barker PB, et al (2014). Clinical proton MR spectroscopy in central nervous system disorders. Radiol, 270, 658-79.

Ozbay PO, Ekinci T, Caltekin MD, et al (2015). Comparative evaluation of the risk of malignancy index scoring systems (1-4) used in differential diagnosis of adnexal masses. Asian Pac J Cancer Prev, 16, 345-9.

Simsek HS, Tokmak A, Ozgu E, et al (2014). Role of a risk of malignancy index in clinical approaches to adnexal masses. Asian Pac J Cancer Prev, 15, 7793-7.

Stanwell P, Russell P, Carter J, et al (2008). Evaluation of ovarian tumors by proton magnetic resonance spectroscopy at three Tesla. Invest Radiol, 43, 745-51.

Swindle P, McCredie S, Russell P, et al (2003). Pathologic characterization of human prostate tissue with proton MR spectroscopy. Radiol, 228, 144-51.

Takeuchi M, Matsuzaki K, Nishitani H (2010). Diffusionweighted magnetic resonance imaging of ovarian tumors: differentiation of benign and malignant solid components of ovarian masses. J Comput Assist Tomogr, 34, 173-6.

Tantipalakorn C, Wanapirak C, Khunamornpong S, et al (2014). IOTA simple rules in differentiating between benign and malignant ovarian tumors. Asian Pac J Cancer Prev, 15, 5123-6.

Thomassin-Naggara I, Bazot M, Darai E, et al (2008). Epithelial ovarian tumors: value of dynamic contrast-enhanced MR imaging and correlation with tumor angiogenesis. Radiol, 248, 148-59.

Thomassin-Naggara I, Toussaint I, Perrot N, et al (2011).
Characterization of complex adnexal masses: value of adding perfusion- and diffusion-weighted MR imaging to conventional MR imaging. Radiol, 258, 793-803. 